\title{
Spectrum of acute clinical characteristics of diagnosed concussions in college athletes wearing instrumented helmets
}

\author{
Clinical article
}

\author{
Ann-Christine Duhaime, M.D., ${ }^{1}$ Jonathan G. Beckwith, M.S., ${ }^{2}$ Arthur C. Maerlender, Ph.D., ${ }^{3}$ \\ Thomas W. McAllister, M.D., ${ }^{4}$ Joseph J. Crisco, Ph.D., ${ }^{5}$ Stefan M. Duma, Ph.D., ${ }^{6}$ \\ P. Gunnar Brolinson, D.O., ${ }^{7}$ Steven Rowson, Ph.D., ${ }^{6}$ Laura A. Flashman, Ph.D., ${ }^{4}$ \\ Jeffrey J. Chu, M.S., ${ }^{2}$ And Richard M. Greenwald, Ph.D.,
}

${ }^{1}$ Department of Neurosurgery, Massachusetts General Hospital, Harvard University, Boston, Massachusetts; ${ }^{2}$ Simbex, Lebanon; ${ }^{3}$ Pediatric Neuropsychological Services and ${ }^{4}$ Department of Psychiatry, Dartmouth Medical School, Lebanon; ${ }^{8}$ Thayer School of Engineering, Dartmouth College, Hanover, New Hampshire; ${ }^{5}$ Bioengineering Laboratory,

Department of Orthopaedics, Warren Alpert Medical School, Brown University and Rhode Island Hospital, Providence, Rhode Island; ${ }^{6}$ Virginia Tech-Wake Forest Center for Injury Biomechanics; and ${ }^{7}$ Edward Via Virginia College of Osteopathic Medicine, Blacksburg, Virginia

Object. Concussive head injuries have received much attention in the medical and public arenas, as concerns have been raised about the potential short- and long-term consequences of injuries sustained in sports and other activities. While many student athletes have required evaluation after concussion, the exact definition of concussion has varied among disciplines and over time. The authors used data gathered as part of a multiinstitutional longitudinal study of the biomechanics of head impacts in helmeted collegiate athletes to characterize what signs, symptoms, and clinical histories were used to designate players as having sustained concussions.

Methods. Players on 3 college football teams and 4 ice hockey teams (male and female) wore helmets instrumented with Head Impact Telemetry (HIT) technology during practices and games over 2-4 seasons of play. Preseason clinical screening batteries assessed baseline cognition and reported symptoms. If a concussion was diagnosed by the team medical staff, basic descriptive information was collected at presentation, and concussed players were reevaluated serially. The specific symptoms or findings associated with the diagnosis of acute concussion, relation to specific impact events, timing of symptom onset and diagnosis, and recorded biomechanical parameters were analyzed.

Results. Data were collected from 450 athletes with 486,594 recorded head impacts. Forty-eight separate concussions were diagnosed in 44 individual players. Mental clouding, headache, and dizziness were the most common presenting symptoms. Thirty-one diagnosed cases were associated with an identified impact event; in 17 cases no specific impact event was identified. Onset of symptoms was immediate in 24 players, delayed in 11, and unspecified in 13. In 8 cases the diagnosis was made immediately after a head impact, but in most cases the diagnosis was delayed (median 17 hours). One diagnosed concussion involved a 30-second loss of consciousness; all other players retained alertness. Most diagnoses were based on self-reported symptoms. The mean peak angular and rotational acceleration values for those cases associated with a specific identified impact were $86.1 \pm$ $42.6 \mathrm{~g}$ (range $16.5-177.9 \mathrm{~g}$ ) and $3620 \pm 2166 \mathrm{rad} / \mathrm{sec}^{2}\left(\mathrm{range} 183-7589 \mathrm{rad} / \mathrm{sec}^{2}\right)$, respectively.

Conclusions. Approximately two-thirds of diagnosed concussions were associated with a specific contact event. Half of all players diagnosed with concussions had delayed or unclear timing of onset of symptoms. Most had no externally observed findings. Diagnosis was usually based on a range of self-reported symptoms after a variable delay. Accelerations clustered in the higher percentiles for all impact events, but encompassed a wide range. These data highlight the heterogeneity of criteria for concussion diagnosis, and in this sports context, its heavy reliance on self-reported symptoms. More specific and standardized definitions of clinical and objective correlates of a "concussion spectrum" may be needed in future research efforts, as well as in the clinical diagnostic arena.

(http://thejns.org/doi/abs/10.3171/2012.8.JNS112298)

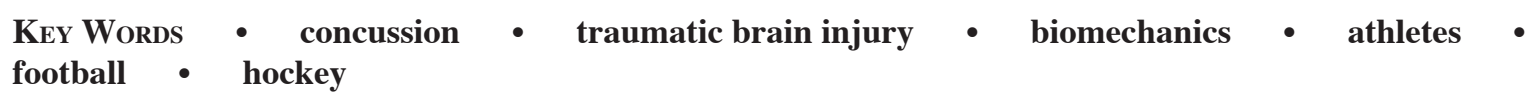

$\mathrm{S}$ PORTS concussions have been the focus of intense attention in recent years, in part related to concerns about catastrophic second-impact syndrome, short-

\footnotetext{
Abbreviations used in this paper: $\mathrm{ATC}=$ certified athletic trainer HIT = Head Impact Telemetry; ImPACT = Immediate Post-Concussion Assessment and Cognitive Test; NCAA = National Collegiate Athletic Association.
}

and long-term neurocognitive effects, and the possible contribution of repeated concussions to delayed brain deterioration..$^{19,20,22,37,40}$ Because of these concerns, more than 26 states to date have enacted legislation requiring students with concussions to be evaluated by appropriately trained personnel before returning to sports participation. However, the definition of concussion has evolved over time and also varies among medical specialties, 
presenting difficulties with knowing exactly which players meet the criteria for this diagnosis and require mandated evaluation. Specifically, the definition has ranged from requiring a loss of consciousness or amnesia for the event, to including varying degrees of alteration in level of consciousness, to including any symptoms such as headache or dizziness after head impact or head motion..$^{2,13,15,32,34,39,43}$ Even in the context of consensus definitions, how diagnostic criteria are applied or ascertained is not always clear and may be changing over time. ${ }^{1}$

Between the fall of 2007 and spring of 2011, our group has studied the biomechanical basis of concussion and the effects of repetitive head impacts on 450 helmeted athletes in 3 NCAA athletic programs (Brown University, Dartmouth College, and Virginia Tech). In this comprehensive investigation, from which the data in the current report are drawn, athletes underwent a clinical screening battery-including measures of symptom reporting, cognition, and, at some sites, balance testing, gene sampling, and neuroimaging - prior to their athletic season, following a diagnosed concussion, and postseason.

The goal of the present study was to analyze the spectrum of clinical presentations that were used by the team medical personnel to make the diagnosis of sports-related concussion within this single multisite study of helmeted college athletes. We sought to characterize the reported histories and signs and symptoms recorded by team medical staff that were used in making a concussion diagnosis, as well as their association with other measured parameters used in this study, including head impact biomechanics. While detailed biomechanical correlates have been published elsewhere or are under review, the specific focus of this report is to characterize the clinical criteria used operationally to diagnose concussion in this study of athletes with repetitive head impacts.

\section{Methods}

A total of 450 athletes who had signed informed consent forms approved by their college's institutional review board participated in the study, which was approved by the Dartmouth, Brown, and Virginia Tech institutional review boards. During the season, athletes participating in contact sports (football, ice hockey) wore instrumented helmets to record their head impact exposure (impact frequency, location, and magnitude) during all organized sessions of play (practices, scrimmages, and games). In the event that a diagnosis of concussion was made, the injured athlete and subject-matched controls from both contact and noncontact sports were subjected to the same clinical examinations. In this communication, we focus only on athletes who were diagnosed by team medical personnel as having a concussion during the seasons under study, and we report on the clinical observations and symptomatology that led to the diagnosis of concussion.

\section{Clinical Assessment and Diagnosis of Concussion}

Because the intent of the larger, overall study was to measure biomechanical correlates of diagnosed concussions in collegiate athletes under contemporary conditions of play, the operational definition of what consti- tutes a concussion was left to the discretion of each team's medical staff (certified athletic trainers [ATCs] and team physicians), according to the guidelines currently in use at that institution during the period of study. Specifically, no attempt was made to influence the recognition, diagnosis, or management of injuries, nor return to play guidelines used, during the study. It was felt that in this way, the most accurate representation of the current state of diagnosis and management would not be altered by the performance of the research.

Each of the 3 institutions had general guidelines already in place at the time of the study for the identification and management of concussion. Certified athletic trainers were present at each session of play. All schools agreed with previously published definitions of concussion as a process affecting the brain induced by traumatic mechanical forces and resulting in the rapid onset of short-lived impairment of neurological function, often accompanied by additional characteristic signs and symptoms. ${ }^{32}$ For the purposes of this study, "diagnosed concussion" was defined as a specific diagnosis of an acute or subacute concussion for an individual player by a member of the team's medical staff, using their standard operational definition in use for that team at the time of the study. Athletes who were identified as or suspected of having a concussion were assessed with standard protocols, as follows.

1. For athletes with suspected concussion diagnosed during a session, the ATC generally assessed the athlete on site immediately and at regular, frequent intervals for the development of mental status abnormalities and/or postconcussive symptoms at rest and also with exertion if appropriate. The exact tools used for the sidelines assessment varied among institutions and between sports, with schools typically using tools derived from standardized symptom checklists, ${ }^{32}$ assessment of answers to specific questions involving orientation and memory, ${ }^{31}$ and additional on-field assessments, such as balance testing. ${ }^{23}$

2. Athletes who reported symptoms after, rather than during, the session were assessed in the training room at the discretion of the ATC, typically using a symptom checklist, brief neurological screening, and in some instances, balance testing.

3. The team medical staff (ATC or physician) used a symptom checklist for each subsequent follow-up assessment, typically until all signs and symptoms were absent at rest and during physical exertion.

4. In addition to these preexisting standard team guidelines, as part of the study protocol, all participants completed a preseason evaluation that included symptom checklists, computerized ImPACT testing and, at one site, more detailed paper and pencil neuropsychological evaluations. ImPACT (Immediate Post-Concussion Assessment and Cognitive Test) is a widely used computerized neuropsychological screening tool for sports-related head impact assessment. ${ }^{25,38}$

\section{Presenting Symptoms and Timing Documentation}

Athletes identified by the medical staff as having suspected injuries were assessed and their findings documented on the sidelines or at the time of clinical presentation by the usual methods employed at each participating 
site; documentation typically included a free-text form or notes describing the events and/or complaints. These trainer assessments were used to collect descriptive data about the specific signs and symptoms exhibited, their timing and relationship to identified contact events, and the time of diagnosis. Some, but not all, trainers documented sidelines checklist results obtained on site; this was not required by the overall study protocol. Additionally, basic demographic and anthropometric data (age, height, weight), history of prior concussion, and date of symptom resolution were provided for the study database.

Once an athlete had been diagnosed as having a concussion, the athlete was instructed per study protocol to report for assessment in the training room within 24-72 hours of the event. Any additional documentation regarding symptom onset and circumstances that was collected during this assessment was reviewed for the study. Using all available on-site and initial training room presentation information, specific data about injury circumstances, symptom type, onset, and diagnosis were analyzed. Follow-up data collected as part of the ongoing larger study (such as serial ImPACT testing, more detailed neuropsychological tests, and/or brain imaging) were not used in the present analysis.

\section{Head Impact Measurement}

During all practices and games, players wore either football (Riddell Sports) or hockey helmets (Easton Stealth S-9, Easton Sports; CCM Vector, Reebok) instrumented with the Head Impact Telemetry (HIT) System (Simbex). The HIT System was designed to record in-vivo acceleration of the head following impact, and both its function and validation have been previously

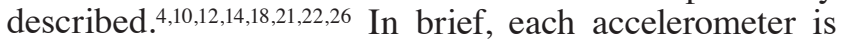
positioned against the head to isolate the head from helmet vibrations, and, when an impact is detected, data are transmitted to a sideline receiver connected to a laptop computer. Accelerometer data recorded from each impact are processed using an optimization algorithm to solve for the linear and rotational acceleration at the head center of gravity and impact location. ${ }^{8}{ }^{89}$ From these available data, descriptive statistics were calculated for athletes in whom concussion was diagnosed; these statistics included the number of impacts sustained on the day of injury and within 7 days of injury, the peak linear and angular acceleration of specific identified impacts associated with a diagnosed concussion, and the location of the specific impacts (separated into 4 general head regions-front, back, top, and side).

\section{Data Element Definitions and Analysis}

To analyze the degree to which concussions were associated with an identified impact event, concussion events were characterized as a) attributed by the player or observers to a specific identified impact (or, in some cases, impact sequence of more than one hit in close temporal proximity, such as head-to-head followed immediately by head-to-ground); b) no specific impact event identified; or c) unknown. For the purposes of the present report, data collected from the helmet accelerometer array were not used to characterize whether a specific identified impact event occurred in association with symptom onset.

To analyze timing of onset of signs and/or symptoms in relation to the impact event, onset of symptoms relative to a causative impact was categorized as a) immediate or near immediate; b) onset delayed, with the specific timing of onset noted; or c) unknown. For purposes of this study, "immediate or near immediate" refers to those events in which the athlete left a session for assessment by the trainer. To further assess the relationship of onset of symptoms relative to impact, the overall group of 48 concussion cases was divided into those that could be associated with a specific impact by either the athlete or observers (31 cases), and those in which the available descriptions state specifically that there was no specific identified impact (13 cases). Four additional diagnosed concussions had insufficient data regarding an identified impact, so these were excluded from this part of the analysis.

Timing of diagnosis was categorized as a) immediate or near immediate; b) delayed, with timing noted; or c) unknown. "Immediate or near immediate" diagnoses included those events in which the player left the session for assessment by the trainer. Delayed diagnoses were those in which ongoing symptoms were brought to the attention of the trainer at a later time, as well as situations in which the player had delayed onset of symptoms.

Mental status was categorized as a) no change; b) subjective change such as "feeling in a fog," trouble with memory, difficulty concentrating, or drowsiness; c) objectively observed by others; or d) unknown. The number of player-reported prior concussions was listed or was designated as unknown.

The methods used to ascertain the clinical characteristics of players in whom concussions were diagnosed in this study are descriptive. More detailed analyses of the biomechanical forces experienced by helmeted athletes, the cognitive correlates of diagnosed concussions as well as the corresponding data from matched controls, and the relationship between these factors have been previously reported or are ongoing. ${ }^{3-5,10,28,35,36}$

\section{Results}

During the 4 years of data collection, 12 team seasons ( 3 teams for 4 seasons) were monitored in football, 6 team seasons ( 2 teams for 3 seasons) in female ice hockey, and 4 team seasons ( 1 team for 3 seasons and 1 team for 1 season) in male ice hockey. A total of 486,594 head impacts were reported from the 450 participating athletes. Fortyeight separate concussions were diagnosed in 44 individual players, with 4 players sustaining 2 concussions each during the study period. Of these diagnosed concussions, 40 were in football, 7 were in women's hockey, and 1 was in men's hockey (Table 1).

\section{Relation to Identified Impact Events}

Thirty-one of 48 diagnosed concussions were associated with an identified impact event by report of the player or observers, while in 13 concussions, no specific impact event could be identified. Four additional events 
TABLE 1: Clinical and biomechanical characteristics of athletes diagnosed with concussion by team medical staff*

\begin{tabular}{|c|c|}
\hline Characteristic & Value \\
\hline \multicolumn{2}{|l|}{ incidence } \\
\hline no. of impacts recorded & 486,594 \\
\hline no. of participating athletes & 450 \\
\hline $\begin{array}{l}\text { no. of diagnosed concussions ( } 40 \text { football, } 7 \text { wom- } \\
\text { en's hockey, } 1 \text { men's hockey) }\end{array}$ & 48 \\
\hline no. of athletes w/ concussion diagnosis & 44 \\
\hline \multicolumn{2}{|l|}{ presenting symptoms/signs } \\
\hline loss of consciousness & 1 \\
\hline mental slowing, confusion, "in a fog" & 36 \\
\hline headache & 29 \\
\hline dizziness & 19 \\
\hline neck pain & 11 \\
\hline head or neck pain only-no change in mentation & 7 \\
\hline \multicolumn{2}{|l|}{ identified impact \& timing of symptom onset } \\
\hline specific impact event identified by player/observers & 31 \\
\hline immediate onset of symptoms & 19 \\
\hline delayed onset of symptoms & 5 \\
\hline unknown onset of symptoms & 7 \\
\hline $\begin{array}{l}\text { no specific impact event identified by player/ } \\
\text { observers }\end{array}$ & 13 \\
\hline immediate onset of symptoms & 5 \\
\hline delayed onset of symptoms & 6 \\
\hline unknown onset of symptoms & 2 \\
\hline \multicolumn{2}{|l|}{ timing of diagnosis } \\
\hline immediate or w/in minutes & 8 \\
\hline later same day & 19 \\
\hline next day & 10 \\
\hline $2-4$ days & 9 \\
\hline \multicolumn{2}{|l|}{ prior concussions } \\
\hline none & 15 \\
\hline 1 & 13 \\
\hline 2 & 4 \\
\hline 3 & 1 \\
\hline unknown & 15 \\
\hline \multicolumn{2}{|l|}{ biomechanics } \\
\hline \multicolumn{2}{|l|}{ peak linear acceleration $(g)$} \\
\hline mean & $86.1 \pm 42.6$ \\
\hline range & $16.5-177.9$ \\
\hline \multicolumn{2}{|l|}{ peak angular acceleration $\left(\mathrm{rad} / \mathrm{sec}^{2}\right)$} \\
\hline mean & $3620 \pm 2166$ \\
\hline range & $183-7589$ \\
\hline
\end{tabular}

* Values refer to concussions unless otherwise indicated.

had insufficient descriptive data to characterize whether a specific impact was correlated with symptom onset (Table 1).

\section{Presenting Symptoms}

The majority of concussions were diagnosed on the basis of subjective complaints voiced to the team medical staff, usually the ATC, and recorded descriptively at the time of diagnosis; most often one or two major symptoms were reported (Table 1). Immediate or delayed symptoms of variable degrees of mental slowing, "clouding," confusion, difficulty concentrating or remembering, or feeling "in a fog" were described at the time of presentation by the athlete in 36 cases. Headache was reported in 29 cases; dizziness in 19; and neck pain in 11. While few players presented to the trainer with stated chief complaints of imbalance, fatigue, irritability, emotional lability, nausea, hearing, or vision problems, these were endorsed with variable frequency in those players for whom symptom checklists were obtained at the time of injury, with "fatigue," "balance difficulty," and "blurred vision" being the most common $(18,15$, and 15 players, respectively). Some athletes presented with complaints using vernacular or nonspecific descriptors such as feeling "messed up," "weird," "out of it," or "not right." Seven athletes in whom concussion was diagnosed reported no change in any aspect of mental status or cognition, having somatic complaints only (for example, headache or neck pain). In 3 athletes there was inadequate information to determine mental status changes.

\section{Observed Signs at Time of Diagnosis}

In addition to subjective reported symptoms, some athletes had witnessed signs of concussion (that is, signs observed by other people), but this occurred in the minority of diagnoses. In one concussion event there was a 30-second loss of consciousness, but all other players retained alertness. In 7 athletes (including the player with loss of consciousness), changes in speech, affect, or mentation were noted by other observers (Table 1).

\section{Timing of Onset of Signs and/or Symptoms}

Nineteen of the 31 diagnosed concussions that were associated with an identified specific impact were characterized by immediate or near-immediate onset of symptoms. In 3 of 31 events, symptoms were specifically described as delayed in onset on the same day (later in the session, after the session, or that night), and in 2 instances the symptoms were first noted on the next day. In an additional 7 diagnosed concussion cases, the timing of onset of symptoms was unknown.

In contrast, of the 13 players with diagnosed concussions without a specific identified single impact, the descriptions of signs and symptoms by the trainers suggested that 5 players had relatively acute symptom onset during play/practice, 6 had delayed symptom onset after play (after session or in subsequent days), and in 2 the timing of symptom onset was unknown (Table 1).

\section{Timing of Diagnosis}

In 2 of the 48 diagnosed concussions, there was insufficient information to estimate time of diagnosis. Of the remaining 46 diagnosed concussions, 8 were diagnosed immediately or within several minutes of the event, and 38 were diagnosed after a delay ranging from 10 minutes to 4 days (mean 23 hours; median 17 hours). Of the 38 
concussions with delay to diagnosis, 19 were diagnosed on the day of the event, 10 were diagnosed on the next day, and 9 were diagnosed between 2 and 4 days after the event (Table 1).

\section{Prior Concussions}

Fifteen players with diagnosed concussions had no prior concussions by self-report, 13 had 1 prior concussion, 4 had 2 prior concussions, 1 had 3 prior concussions, and in 15 players the prior concussion status was unknown.

\section{Biomechanical Parameters of Diagnosed Concussions}

For those players with diagnosed concussions associated with a specific impact event, the mean peak linear acceleration was $86.1 \pm 42.6 \mathrm{~g}$ (range 16.5-177.9 $g$ ) and the mean peak angular acceleration was $3620 \pm 2166 \mathrm{rad} / \mathrm{sec}^{2}$ (range 183-7589 rad $/ \mathrm{sec}^{2}$ ) (Table 1). Impacts to the front of the head $(n=13)$ accounted for the most impacts associated with diagnosed concussion, followed by the top $(n=9)$, back $(n=8)$, and side $(n=5)$.

The total number of impacts on the day of injury and in the previous week were available for 35 of the 48 instances of diagnosed concussion; the respective mean values were $19.5 \pm 15.7$ (for number of head impacts on the day of injury, range 2-53) and $49.7 \pm 43.7$ (for the number of impacts within 7 days of injury, range 4-181).

\section{Discussion}

The main findings in this report were that many players had delayed onset of symptoms after an impact event, the majority of players had delayed diagnosis (delays of hours to days), more than a third of diagnosed concussions were not associated with a specific impact event, and biomechanical correlates, while averaging at the high end of the spectrum, showed significant variability. These observations may raise questions about whether current diagnostic methods and operational definitions of concussion in the context of athletics, based largely on self-report of specific clinical symptoms, capture those individuals actually at risk for short-, intermediate-, and long-term consequences of injury. The findings also point out the differences between the diagnosis of concussion in the context of sport and the cases typically seen by consulting neurosurgeons in emergency departments, in which a single clearly identified event is associated with immediate onset of symptoms including alteration in mental status. The potential consequences of these differences for medical decision making and for further research are explored further below.

\section{Concussion Definitions}

Although concussion has been the focus of a great deal of recent attention, the entity has been difficult to define, both operationally and with respect to underlying pathophysiology. Clinical criteria for the diagnosis of concussion have varied over time and among specialties. Historically, concussion was defined as a brief loss of consciousness sustained after head impact. ${ }^{13,42}$ These clinical events could be demonstrated by large-animal modeling to occur at the lower magnitudes of a spectrum of angular deceleration, which, at higher magnitudes, leads to diffuse axonal injury and prolonged coma. ${ }^{15,34}$ Theories of why consciousness is impaired after head impact have included vascular, reticular, centripetal, pontine cholinergic, and convulsive hypotheses, but the actual situation in vivo remains incompletely understood. ${ }^{39}$

The definition of concussion later was widened to include transient alterations in consciousness without actual loss of consciousness, with the recognition that similar postconcussive symptoms, including amnesia, could occur in both scenarios. ${ }^{43}$ The relatively recent attention to sports injuries has contributed to further evolution of operational criteria used to make a concussion diagnosis. In part because of concerns about under-recognition, consensus conferences such as the Zurich Conference formulated a new definition, which included any type of alteration in mentation, such as confusion, disorientation, or mental clouding, as opposed to relying specifically on loss of consciousness or amnesia. ${ }^{32}$

Studies of series of patients with concussion have grouped postconcussive complaints into categories, including somatic, emotional, cognitive, and sleep. ${ }^{16,17} \mathrm{Op}-$ erational definitions of concussion used by some sports personnel more recently have included the occurrence of any of these types of symptoms, whether any specific loss of consciousness, amnesia, or alteration of mentation occurs. Thus, a player with, for instance, transient headache or dizziness only, may be diagnosed as having a concussion in some contexts. While broadening the scope of symptoms used to make a diagnosis, these approaches have not specifically addressed variability in timing of onset of symptoms, nor do they guide the clinician in how to handle the situation in which symptoms are reported without being associated with a specifically identified impact event. This has led to confusion on the part of coaches and trainers, parents, and clinicians, with respect to what exactly constitutes a concussion and who needs to be referred for evaluation.

\section{Variability in Concussion Diagnostic Criteria}

The data collected in this study reflect diagnostic criteria used for concussion in the context of collegiate sports, which may have unique characteristics compared with other medical settings for concussion diagnosis. In this study, while athletes typically sustained multiple impacts on the day of injury, only about two-thirds of diagnosed concussions were attributed to a specific impact event by the player or team staff. A minority of diagnosed concussions had objective neurological signs including only one with loss of consciousness, and 7 players had no mental status changes. While half of players with concussion had immediate onset of symptoms, many specifically reported delayed symptom onset, and more than $80 \%$ of concussion diagnoses were made after a delay, in some cases up to several days. Despite this variability, symptoms did cluster along the lines described by previous researchers, with a preponderance of subjective transient alteration in cognition and headache. ${ }^{24}$ The operational diagnosis of concussion used in the sports setting may not reflect the same injury spectrum seen by neurosurgeons 
in an emergency department or hospital, where a single impact event associated with clear loss of consciousness is more likely to be the reason to obtain neurosurgical consultation. This may result in potential differences between disciplines in patient populations described by the same terminology.

\section{Variability in Biomechanical Correlates}

For those diagnosed concussions that were attributed to a specific contact event by the athlete or observers, the measured accelerations in the present study clustered at the high end of the spectrum seen in these sports overall, but considerable variability was encountered. The mean peak linear and angular accelerations for contact events associated with diagnosed concussion (Table 1) were greater than the 95th percentile for peak linear acceleration reported previously in football $\left(62.7 \mathrm{~g}\right.$ and $\left.2975 \mathrm{rad} / \mathrm{sec}^{2}\right)$ and for hockey (43.7g and $4674 \mathrm{rad} / \mathrm{sec}^{2}$ for males; $44.9 g$ and 3709 $\mathrm{rad} / \mathrm{sec}^{2}$ for females). ${ }^{5,11}$ However, there were a number of contact events associated with a diagnosis of concussion that demonstrated considerably lower accelerations. Conversely, many players had similar or higher accelerations recorded and did not report symptoms or have neurological signs reported by other observers. ${ }^{11}$

The variability in measured accelerations in players with diagnosed concussions might reflect a number of factors. First, multiple biomechanical variables exist (number of prior impacts, linear acceleration, rotational acceleration, impact location, impact duration, and plane of rotation), each of which may influence injury, and the combination of these factors may result in different clinical presentations. Prior impact history may precondition the brain to development of subsequent symptoms with additional lower magnitude accelerations, or, conversely, may be responsible for meliorated or delayed symptoms. Variability in symptom reporting among players may limit correlation between measured accelerations and symptoms. Host factors including neuroanatomical, vascular, maturational, and genetic factors affecting injury response and repair may influence the experience of specific signs or symptoms. Additionally, while head kinematics obtained from the HIT System can be combined with finite element models to obtain estimates of brain tissue motion following impact, there is at present no technology available that can measure actual strains experienced by the brain in vivo, which may vary despite similar head accelerations..$^{30,41}$ Finally, while it has been demonstrated that head impact kinematics obtained from the HIT System are highly correlated with laboratory surrogates, measurements for any single impact should be assumed to contain error dependent on both the measure of interest and impact conditions. ${ }^{4}$ More detailed analysis of these biomechanical factors and their relationship to clinical signs and symptoms using data from the helmet accelerometer studies is ongoing. ${ }^{3,28,30,36}$

\section{Implications for Prevention and Intervention Trials}

The rationale for accurately diagnosing "concussion" is to adequately prevent and manage both short-term and more long-term consequences of injury. Concerns over acute impairments after head injury that could put performance at risk both in sports and in the military have led to the development of a variety of strategies to enhance immediate diagnosis, ranging from sideline cognitive tests to sophisticated neurophysiological and biomarker measures. ${ }^{16,27}$ Return to play after unrecognized hemorrhage or vasomotor instability is thought to increase the risk of the rare but highly morbid second-impact syndrome, which can occur over an acute to intermediate time frame, up to several weeks after initial injury. ${ }^{7,37}$ Likewise, exacerbation of cognitive dysfunction due to potentially synergistic effects of repeated injuries during this intermediate time frame has been hypothesized. ${ }^{6,29}$ More recently, it has been suggested that mechanical trauma may begin a chain of events, at least in some susceptible individuals, leading to more chronic changes, including psychiatric disorders and dementia. ${ }^{33}$

One of the main goals of diagnosis of clinical or subclinical brain dysfunction is to prevent those events that can cause acute, intermediate, and/or long-term harm. Historically, diagnosis has rested on specific clinical signs and symptoms, but these may be unreliable indicators of actual cellular, subcellular, or vascular injury or predictors of subsequent adverse consequences after head impact events. It is possible that deleterious pathophysiological cascades are initiated even when signs and symptoms are absent. Conversely, some symptoms may not, in fact, predict increased risk for specific deleterious consequences. Thus, different symptoms may reflect different networks or processes, each of which may expose individuals to specific acute or delayed risks, and may have different temporal profiles for repair and resolution. Current use of return to baseline neurocognitive scores, along with lack of signs or symptoms at rest and exertion, as a gate for return to play has not yet been validated as an indicator that the various potential risks of adverse consequences from return to play have passed. It is possible that technological adjuncts that can measure mechanical loads, biomarker arrays, cerebrovascular reactivity, or other parameters may be better predictors of risk and prognosis than are clinical signs and symptoms.

Treatment trials also rely on appropriate prediction of risk factors for poor outcome and on identifying interventions that can improve on current management. If a trial includes both patients who are at high risk for an outcome and those who have minimal risk, the treatment effect will be diluted, and potential efficacy difficult to discern.

For all these reasons, the single term "concussion" as currently used in clinical practice may encompass such a wide spectrum of entities that it may be of limited use in furthering understanding of traumatic brain dysfunction/ injury at the milder end of the severity spectrum. Having better descriptive and diagnostic tools to characterize these events, to predict specific outcome parameters, to prevent reinjury, and to stratify treatment trials would be welcome, and likely would involve refinement in clinical definitions as well as the use of adjunctive diagnostic tests. A more encompassing term such as "concussion spectrum" may reinforce the idea that specific characterization tools for individual patients still need to be defined to reflect potentially different pathophysiological 
entities and their various associated consequences. It is conceivable that multiple repetitive head contacts, such as those analyzed in this study, have a significantly different pathophysiological and/or temporal profile compared with the single-event, loss-of-consciousness concussion seen very commonly in emergency departments. These data also suggest that at the present time, the presence or absence of concussion "symptoms" cannot be strictly equated with the presence or absence of cellular, subcellular, or neurovascular injury or damage to the brain.

\section{Conclusions}

This study demonstrates the wide variety of presenting symptoms, timing of symptom onset and diagnosis, and biomechanical correlates of diagnosed concussion as defined in contemporary collegiate sports. Many players had delayed onset of symptoms, and most had delayed diagnosis. It remains unclear to what extent these various clinical criteria actually predict acute, intermediate, and long-term risks after mechanical loading of the head, compared with the risks in players with similar mechanical loads who do not experience or report symptoms. Further work is ongoing to determine whether specific clinical profiles, biomechanical parameters, test scores, imaging features, or genetic characteristics best predict those patients at risk for adverse consequences from participation in contact sports.

\section{Disclosure}

Richard M. Greenwald, Jeffrey Chu, and Joseph J. Crisco and Simbex have a financial interest in the instruments (HIT System, Sideline Response System, Riddell, Inc.) that were used to collect the biomechanical data reported in this study. Mr. Beckwith is an employee of Simbex.

The research for this study is supported by the National Institute for Child Health and Human Development at the National Institutes of Health (Grant R01HD048638).

Author contributions to the study and manuscript preparation include the following. Conception and design: Duhaime, Beckwith, Maerlender, McAllister, Crisco, Duma, Brolinson, Chu, Greenwald. Acquisition of data: all authors. Analysis and interpretation of data: Duhaime, Beckwith, Maerlender, McAllister, Crisco, Chu, Greenwald. Drafting the article: Duhaime, Beckwith, Maerlender, Greenwald. Critically revising the article: all authors. Reviewed submitted version of manuscript: all authors. Approved the final version of the manuscript on behalf of all authors: Duhaime. Administrative/ technical/material support: all authors. Study supervision: Duhaime, Beckwith, Maerlender, McAllister, Crisco, Duma, Brolinson, Greenwald.

\section{References}

1. Alla S, Sullivan SJ, McCrory P: Defining asymptomatic status following sports concussion: fact or fallacy? Br J Sports Med 46:562-569, 2012

2. Aubry M, Cantu RC, Dvorak J, Graf-Baumann T, Johnston K, Kelly J, et al: Summary and agreement statement of the First International Conference on Concussion in Sport, Vienna 2001. Recommendations for the improvement of safety and health of athletes who may suffer concussive injuries. Br J Sports Med 36:6-10, 2002

3. Beckwith JG, Chu JJ, McAllister TW, Maerlender AC, Flashman LA, Duhaime AC, et al: Neurocognitive function and the severity of head impacts sustained in athletic competition. Presented at the Eighth World Congress on Brain Injury, Washington, DC, 2010 (Abstract) (https://ibia .conferenceservices.net/reports/template/onetextabstract.xml?xsl= template/onetextabstract.xsl\&conferenceID=1677\&abstractID =352691) [Accessed August 23, 2012]

4. Beckwith JG, Greenwald RM, Chu JJ: Measuring head kinematics in football: correlation between the head impact telemetry system and Hybrid III headform. Ann Biomed Eng 40:237-248, 2012

5. Brainard LL, Beckwith JG, Chu JJ, Crisco JJ, McAllister TW, Duhaime AC, et al: Gender differences in head impacts sustained by collegiate ice hockey players. Med Sci Sports Exerc 44:297-304, 2011

6. Cantu RC: Cerebral concussion in sport. Management and prevention. Sports Med 14:64-74, 1992

7. Cantu RC, Mueller FO: Brain injury-related fatalities in American football, 1945-1999. Neurosurgery 52:846-853, 2003

8. Chu JJ, Beckwith JG, Crisco JJ, Greenwald RM: A novel algorithm to measure linear and rotational acceleration using single-axis accelerometers. J Biomech 39 (Suppl 1):S534, 2006 (Abstract)

9. Crisco JJ, Chu JJ, Greenwald RM: An algorithm for estimating acceleration magnitude and impact location using multiple nonorthogonal single-axis accelerometers. J Biomech Eng 126:849-854, 2004

10. Crisco JJ, Fiore R, Beckwith JG, Chu JJ, Brolinson PG, Duma $\mathrm{S}$, et al: Frequency and location of head impact exposures in individual collegiate football players. J Athl Train 45:549559,2010

11. Crisco JJ, Wilcox BJ, Machan JT, McAllister TW, Duhaime AC, Duma SM, et al: Magnitude of head impact exposures in individual collegiate football players. J Appl Biomech 28: 174-183, 2012

12. Duma S, Manoogian S, Brolinson PG, Goforth M, Donnenwerth J, Greenwald RM, et al: Measuring real time head accelerations in collegiate football players. Presented at the 2004 Annual Meeting of the American Society of Biomechanics, Portland, OR, 2004 (Abstract) (http://www.asbweb.org/ conferences/2004/pdf/225.pdf) [Accessed August 23, 2012]

13. Fisher CM: Concussion amnesia. Neurology 16:826-830, 1966

14. Funk JR, Duma SM, Manoogian SJ, Rowson S: Biomechanical risk estimates for mild traumatic brain injury. Annu Proc Assoc Adv Automot Med 51:343-361, 2007

15. Gennarelli TA: Head injury in man and experimental animals: clinical aspects. Acta Neurochir Suppl (Wien) 32:1-13, 1983

16. Gioia GA, Collins M, Isquith PK: Improving identification and diagnosis of mild traumatic brain injury with evidence: psychometric support for the acute concussion evaluation. J Head Trauma Rehabil 23:230-242, 2008

17. Gioia GA, Schneider JC, Vaughan CG, Isquith PK: Which symptom assessments and approaches are uniquely appropriate for paediatric concussion? Br J Sports Med 43 (1 Suppl 1): $i 13-i 22,2009$

18. Greenwald RM, Gwin JT, Chu JJ, Crisco JJ: Head impact severity measures for evaluating mild traumatic brain injury risk exposure. Neurosurgery 62:789-798, 2008

19. Guskiewicz KM, Marshall SW, Bailes J, McCrea M, Cantu $\mathrm{RC}$, Randolph C, et al: Association between recurrent concussion and late-life cognitive impairment in retired professional football players. Neurosurgery 57:719-726, 2005

20. Guskiewicz KM, Marshall SW, Bailes J, McCrea M, Harding HP Jr, Matthews A, et al: Recurrent concussion and risk of depression in retired professional football players. Med Sci Sports Exerc 39:903-909, 2007

21. Gwin JT, Chu JJ, Greenwald RM: Head impact telemetry system for measurement of head acceleration in ice hockey. $\mathbf{J}$ Biomech 39 (Suppl 1):S153, 2006 (Abstract) 
22. Gwin JT, Chu JJ, McAllister TW, Greenwald RM: In situ measures of head impact acceleration in NCAA Division 1 men's ice hockey: implications for ASTM F1045 and other ice hockey helmet standards. J ASTM Int 6:1-10, 2009

23. Lovell MR, Collins MW: Neuropsychological assessment of the college football player. J Head Trauma Rehabil 13:9-26, 1998

24. Macciocchi SN, Barth JT, Alves W, Rimel RW, Jane JA: Neuropsychological functioning and recovery after mild head injury in collegiate athletes. Neurosurgery 39:510-514, 1996

25. Maerlender A, Flashman L, Kessler A, Kumbhani S, Greenwald R, Tosteston T, et al: Examination of the construct validity of ImPACT computerized test, traditional, and experimental neuropsychological measures. Clin Neuropsychol 24: $1309-1325,2010$

26. Manoogian S, McNeely D, Duma S, Brolinson G, Greenwald RM: Head acceleration is less than 10 percent of helmet acceleration in football impacts. Biomed Sci Instrum 42:383-388, 2006

27. Marion DW, Curley KC, Schwab K, Hicks RR: Proceedings of the military mTBI Diagnostics Workshop, St. Pete Beach, August 2010. J Neurotrauma 28:517-526, 2011

28. McAllister TW, Flashman LA, Maerlender A, Greenwald RM, Beckwith JG, Tosteson TD, et al: Cognitive effects of one season of head impacts in a cohort of collegiate contact sport athletes. Neurology 78:1777-1784, 2012

29. McAllister TW, Flashman LA, McDonald, Saykin AJ: Mechanisms of working memory impairment after mild and moderate TBi: evidence from functional MRI and neurogenetics. J Neurotrauma 23:1450-1467, 2006

30. McAllister TW, Ford JC, Ji S, Beckwith JG, Flashman LA, Paulsen K, et al: Maximum principal strain and strain rate associated with concussion diagnosis correlates with changes in corpus callosum white matter indices. Ann Biomed Eng 40:127-140, 2012

31. McCrea M, Kelly JP, Randolph C, Kluge J, Bartolic E, Finn $\mathrm{G}$, et al: Standardized assessment of concussion (SAC): onsite mental status evaluation of the athlete. J Head Trauma Rehabil 13:27-35, 1998

32. McCrory PR, Meeuwisse W, Johnston K, Dvorak J, Aubry M, Molloy M, et al: Consensus statement on Concussion in Sport-the 3rd International Conference on Concussion in Sport held in Zurich, November 2008. J Sci Med Sport 12: 340-351, 2009

33. McKee AC, Cantu RC, Nowinski CJ, Hedley-Whyte ET, Ga- vett $\mathrm{BE}$, Budson $\mathrm{AE}$, et al: Chronic traumatic encephalopathy in athletes: progressive tauopathy after repetitive head injury. J Neuropathol Exp Neurol 68:709-735, 2009

34. Ommaya AK, Gennarelli TA: Cerebral concussion and traumatic unconsciousness. Correlation of experimental and clinical observations of blunt head injuries. Brain 97:633-654, 1974

35. Rowson S, Duma SM: Development of the STAR evaluation system for football helmets: integrating player head impact exposure and risk of concussion. Ann Biomed Eng 39:2130 2140, 2011

36. Rowson S, Duma SM, Beckwith JG, Chu JJ, Greenwald RM, Crisco JJ, et al: Rotational head kinematics in football impacts: an injury risk function for concussion. Ann Biomed Eng 40:1-13, 2012

37. Saunders RL, Harbaugh RE: The second impact in catastrophic contact-sports head trauma. JAMA 252:538-539, 1984

38. Schatz P, Pardini JE, Lovell MR, Collins MW, Podell K: Sensitivity and specificity of the ImPACT Test Battery for concussion in athletes. Arch Clin Neuropsychol 21:91-99, 2006

39. Shaw NA: The neurophysiology of concussion. Prog Neurobiol 67:281-344, 2002

40. Silver JM, McAllister TW, Arciniegas DB: Depression and cognitive complaints following mild traumatic brain injury. Am J Psychiatry 166:653-661, 2009

41. Takhounts EG, Ridella SA, Hasija V, Tannous RE, Campbell JQ, Malone D, et al: Investigation of traumatic brain injuries using the next generation of simulated injury monitor (SIMon) finite element head model. Stapp Car Crash J 52:1-31, 2008

42. Valadka AB, Narayan RK: Emergency room management of the head-injured patient, in Narayan RK, Wilberger JE Jr, Povlishcok JT (eds): Neurotrauma. New York: McGraw-Hill, 1996, pp 119-135

43. Yarnell PR, Lynch S: The 'ding': amnestic states in football trauma. Neurology 23:196-197, 1973

Manuscript submitted January 4, 2012.

Accepted August 21, 2012.

Please include this information when citing this paper: published online October 2, 2012; DOI: 10.3171/2012.8.JNS112298.

Address correspondence to: Ann-Christine Duhaime, M.D., Pediatric Neurosurgery, Massachusetts General Hospital, 15 Parkman Street, Wang 331, Boston, Massachusetts 02114. email: aduhaime@ partners.org. 